\title{
Nonexistence of the Classical Trajectories in the Stern-Gerlach Experiment
}

\author{
M. ArseniJeviĆ* AND M. Dugić \\ Department of Physics, Faculty of Science, R. Domanovića 12, 34000 Kragujevac, Serbia
}

\begin{abstract}
The Stern-Gerlach experiment is a paradigm of the quantum measurement of spin. Its physical interpretation is in intimate relation with the physical basis of the current research in the atomic (molecular) nanofabrication procedures. Nevertheless, interpretation of the experiment is an open issue yet. Here, we give the arguments for the physical nonexistence of the so-called classical trajectories of the atoms (molecules) in front of the screen. Some nanotech-related consequences are distinguished.
\end{abstract}

PACS numbers: 03.65.Ta, 03.65.Yz, 81.16.Ta

\section{Introduction}

The Stern-Gerlach experiment is a paradigm of quantum measurement of a particle's spin. However, the deeper physical description of the Stern-Gerlach (SG) effect is an open issue.

Historically the first, and often used, interpretation (probably due to Bohr, Mott and Pauli [1]) of the experiment reads: the atoms behind the Stern-Gerlach magnet possess the definite, physically objective (classical) trajectories. This picture (interpretation) supports the semiclassical description in the terms of the external force for the atom in the external magnetic field $\boldsymbol{B}$. The screen capturing the incident atoms is assumed simply to record the objectively existing trajectories - very much like a camera records of the life scenes. As the quantum apparatus, the screen plays the passive role in the quantum measurement of spin. This interpretation may suggest the universal existence of the atomic trajectories, thus setting a specific basis for the manipulation with the atomic beams. Formally, the state of an ensemble of atoms can be described by a mixed state of the following (simplified) form:

$$
\begin{aligned}
& \hat{\rho}_{\mathrm{S}+\mathrm{CM}}=\frac{1}{2} \\
& \quad \times\left(|-\rangle_{\mathrm{CM}}\langle-|\otimes| \uparrow\rangle_{\mathrm{S}}\langle\uparrow|+|+\rangle_{\mathrm{CM}}\langle+|\otimes| \downarrow\rangle_{\mathrm{S}}\langle\downarrow|\right),
\end{aligned}
$$

where $|+\rangle_{\mathrm{CM}}$ and $|-\rangle_{\mathrm{CM}}$ are the states of the center-of-mass system (up and down trajectories, respectively) and $|\downarrow\rangle_{\mathrm{S}}$ and $|\uparrow\rangle_{\mathrm{S}}$ are the eigenstates of the spin-1/2 projection along the axis of the external field. The mixed state Eq. (1) is virtually the most general mixed state that provides the (required) correlations between the pure states $| \pm\rangle_{\mathrm{CM}}$ and $|\uparrow\rangle_{\mathrm{S}}$, while admitting the inter-

\footnotetext{
* corresponding author; e-mail: momirarsenijevic@kg.ac.rs
}

pretation in the terms of the definite atomic trajectories. Certainly, the manipulation with the atomic trajectories is classical for the system in the state described by Eq. (1).

However, the standard, and generally used model of the Stern-Gerlach experiment [2] does not support this interpretation, neither positively rejects it. The physical situation is described by the following Hamiltonian [2]:

$$
\hat{H}=\frac{\hat{\boldsymbol{P}}_{\mathrm{CM}}^{2}}{2 m} \otimes \hat{I}_{\mathrm{S}}+\mu B\left(\hat{z}_{\mathrm{CM}}\right) \otimes \hat{\sigma}_{\mathrm{S} z}
$$

where $\hat{\boldsymbol{P}}_{\mathrm{CM}}$ and $\hat{z}_{\mathrm{CM}}$ refer to the atomic center-of-mass momentum and position observables, respectively, $B\left(\hat{z}_{\mathrm{CM}}\right)$ stands for the classical magnetic field, while $\hat{\sigma}_{\mathrm{S} z}$ denotes the $z$-component of the spin operator, i.e. its projection along the $z$-axis ( $\mu$ and $m$ have the standard meaning of the Bohr's magneton and of the mass of the atom, respectively). The Hamiltonian Eq. (2) gives rise to the entangled pure state for the composite system [2] of the (simplified) form (cf. e.g. [3] for some details):

$$
|\Psi\rangle_{\mathrm{S}+\mathrm{CM}}=\frac{1}{\sqrt{2}}\left(|\uparrow\rangle_{\mathrm{S}}|-\rangle_{\mathrm{CM}}+|\downarrow\rangle_{\mathrm{S}}|+\rangle_{\mathrm{CM}}\right) .
$$

Needless to say, the entangled state Eq. (3) suggests the substantially different physical picture and the system-manipulation than the state Eq. (1). The state Eq. (3) bears quantum nonseparability and nonlocality, the prominent quantum information resources [4]; formally, the state Eq. (3) is a pair-of-qubits state [3]. The presence of nonlocality/nonseparability in the state Eq. (3) opens the (classically nonexisting [4]) possibilities for manipulating the atomic CM system. E.g., by manipulating the atomic spin-system S, one can manipulate the atomic CM system [4]. In this model, the screen plays an active role as the quantum apparatus - it induces the transition of the $\mathrm{CM}+\mathrm{S}$ state from Eq. (3) to Eq. (1), thus providing the classical information about the atomic trajectory. 
It is worth noting: the states Eq. (1) and Eq. (3) represent the idealizations of the realistic quantum states. Actually, the realistic physical situations are described by the time dependent states that, in turn, makes the task of designing an experimental situation for distinguishing the pure and mixed states for $\mathrm{CM}+\mathrm{S}$ system a subtle task, indeed. Bearing this in mind, a theoretical analysis might be useful for providing the conclusion about the physical existence of the classical trajectories.

In this paper, we perform an ab initio, the decoherence-theory-based [5] analysis of the Stern-Gerlach experiment. Without resorting to any interpretation whatsoever, we are able to draw the definite conclusion on the nonexistence of the classical trajectories. This conclusion provides some physical rewards of interest for the foundations of quantum mechanics (e.g. for the quantum measurement theory) as well in principle as for the advent of the atomic (molecular) nanofabrication (cf., e.g. [6, 7]) by setting the general recipes for manipulation with the atomic (molecular) trajectories.

\section{The decoherence-based model of the SG experiment}

The universally valid quantum mechanics states: the existence of the classical trajectories (as described by Eq. (1)) requires some external action exerted on the system of interest $\mathrm{CM}+\mathrm{S}[5,8]$. Such systems are referred to as open quantum systems, as distinct from the closed (isolated) systems that are subject to the Schrödinger law. The open systems cannot be described by the Schrödinger equation, while robustness (dynamical stability) of certain states of the system (such as the supposed atomic trajectories) is induced by the system's environment. Without the environment, the system (here, the composite system $\mathrm{CM}+\mathrm{S}$ ) is both described by the Schrödinger equation and can be found in superposition of states (such as the entangled state Eq. (3)). Therefore, our starting point in the search for the classical trajectories, Eq. (1), is the openness of the system $\mathrm{CM}+\mathrm{S}$ (more precisely: of the system $\mathrm{CM}$ ) that appears as a hypothesis of our approach. To this end, we employ the socalled environment-induced-decoherence theory that provides existence of the robust open-system's states on the fundamental quantum mechanical level $[5,8]$. The robust states are robust relative to the influence of the environment and thus give rise to the approximately classical behavior of the open system.

A decoherence-based model has recently been proposed [9]. The model describes a free atom exposed in the vacuum to the external classical magnetic field. The model minimally extends the standard model of the SG experiment [2] by stipulating existence of the atomic CM-system environment $\mathrm{R}$ possibly capable of inducing decoherence for certain states of the atomic CM system. Physically, the system $R$ is supposed to represent the set of the relative positions (the internal degrees of freedom of an atom) for the particles constituting the atom.
A proposal for the experimental test of the model has also been given [9]. However, the model is purely qualitative -the existence of the environment $\mathrm{R}$ is stipulated - and the more elaborate analysis is needed. Particularly, the model raises the following question: whether or not one can recognize/construct the realistic internal environment $\mathrm{R}$ for the atomic $\mathrm{CM}$ system?

In order to obtain an interpretation-free answer to this question, we perform an ab initio analysis of the Stern-Gerlach experiment. We deal with the fundamental (yet nonrelativistic) model of atom, and we search for the realistic physical model of the internal environment $R$. We do not employ any additional stipulations and/or interpretations, thus setting a basis for the definite answer to the question of whether or not the classical trajectories in the SG experiment can be considered to be physically realistic.

\subsection{The atomic center-of-mass}

Typically, the internal environment is modeled as a thermal bath or as a collection of the (not necessarily thermalized) harmonic oscillators [10, 11]. However, as we repeatedly emphasize: throughout this paper, we perform an $a b$ initio analysis of the system of interest, without resorting to such formal models. In other words: our task is to recognize the realistic environment for the atomic center-of-mass system. For this purpose, we start from the fundamental (yet nonrelativistic) definition of an atom as a collection of the electrons, protons and neutrons $(\mathrm{e}+\mathrm{p}+\mathrm{n})$ - for the point-like nucleus (as typical for atomic physics, that we do not deal with), the protons + neutrons represents simply the point-like atomic nucleus, $\mathrm{N}(p+n=N)$. Then the atom (while neglecting the atomic spin) is defined by the following Hamiltonian:

$$
\begin{aligned}
\hat{H} & =\sum_{i=1}^{Z} \hat{T}_{\mathrm{e} i}+\sum_{j=1}^{Z} \hat{T}_{\mathrm{p} j}+\sum_{k=1}^{A-Z} \hat{T}_{\mathrm{n} k} \\
& +\hat{V}_{\text {Coul }}^{\mathrm{ee}}+\hat{V}_{\text {Coul }}^{\mathrm{ep}}+\hat{V}_{\text {Coul }}^{\mathrm{pp}}+\hat{V}_{\mathrm{nucl}},
\end{aligned}
$$

where $\hat{T}$ stands for the kinetic terms, $\hat{V}_{\text {Coul }}$ for the Coulomb interaction for the pairs of particles (ee - the electrons, ep - the electron-proton, pp - the protons pairs), while $\hat{V}_{\text {nucl }}$ stands for the nuclear interaction.

The expression Eq. (1) distinguishes the physical importance of the atomic center of mass (CM) subsystem as the system of interest. Then, as it is well known, the set of the particles $\mathrm{e}+\mathrm{p}+\mathrm{n}$ can be redefined as $\mathrm{CM}+\mathrm{R}$, where $\mathrm{CM}$ and $\mathrm{R}$ represent the atomic center-of-mass and the relative particle subsystems. Then, the Hamiltonian Eqs. (4), (2) obtain the following form:

$$
\hat{H}=\hat{H}_{\mathrm{CM}}+\hat{H}_{\mathrm{R}}+\hat{H}_{\mathrm{CM}+\mathrm{S}},
$$

where $\hat{H}_{\mathrm{CM}} \equiv \hat{T}_{\mathrm{CM}}$ is the kinetic term of the atomic center-of-mass, while the R-system's Hamiltonian reads $\hat{H}_{\mathrm{R}}=\sum_{\alpha=1}^{A+Z-1} \hat{T}_{\mathrm{R}_{\alpha}}+\hat{V}_{\text {nucl }}^{(R)}+\hat{V}_{\text {Coul }}^{(R)}+\hat{M}_{\rho \sigma}^{(R)}$, and $\hat{M}_{\rho \sigma}^{(R)}$ represents the internal interaction. As absent from the standard model [2] of the Stern-Gerlach experiment, the subsystem $\mathrm{R}$ may be expected to play the role of the CM's environment [9]. 
From Eq. (5), it is rather apparent: the atomic CM and $\mathrm{R}$ subsystems appear mutually exactly decoupled thus representing the noninteracting systems. Needless to say, then there is no room for the atomic $\mathrm{R}$ system to play the atomic CM system's environment. Formally, as it can be easily shown, the state of the composite system $\mathrm{CM}+\mathrm{S}+\mathrm{R}$ is of the separable form $|\Psi\rangle_{\mathrm{CM}+\mathrm{S}}|\phi\rangle_{\mathrm{R}}$, where $|\Psi\rangle_{\mathrm{CM}+\mathrm{S}}$ is presented by Eq. (3) - there are not the classical trajectories.

It is worth noting: the proper external actions can induce the coupling between the $\mathrm{CM}$ and $\mathrm{R}$ subsystems of an atom $[6,7]$, or even can induce the appearance of the classical trajectories for the atom [12]. However, we do not assume any external action exerted on the atom that might produce such consequences. Rather, we deal with the isolated atom traversing in the vacuum the SG magnet, thus removing the possibility of the appearance of the classical trajectories, except (and only) due to the influence of the internal environment $\mathrm{R}$.

\subsection{The nucleus' center-of-mass}

While this argument and conclusion are as simple and clear as they are, the following remark is also in order. More than $99 \%$ of the atomic mass is placed in the atomic nucleus. Due to the virtual indistinguishability of the atomic and the atomic-nucleus center-of-mass systems, one may wonder if the Stern-Gerlach experiment should be expressed in the terms of the later. For this reason, and for simplicity, we redefine the atomic system as follows: leaving the electrons system intact, we introduce the nucleus $\mathrm{CM}$ and $\mathrm{R}$ subsystems (the use of the same notation as for the atomic $\mathrm{CM}$ and $\mathrm{R}$ system should not introduce any confusion). Now, we ask if there may be any interaction between the nucleus' $\mathrm{CM}$ and $\mathrm{R}$ subsystems.

Now, the atom is formally defined as e $+\mathrm{CM}+\mathrm{R}$; for the point-like nucleus, the atom is simply e + CM (i.e. $\mathrm{CM} \equiv \mathrm{N})$. Then the Hamiltonian $\hat{H}$, Eq. (4), reads:

$$
\begin{aligned}
\hat{H} & =\hat{H}_{\mathrm{e}}+\hat{H}_{\mathrm{CM}}+\hat{H}_{\mathrm{R}} \\
& +\sum_{i, j=1}^{Z} \frac{k}{\left|\hat{\boldsymbol{r}}_{\mathrm{e}_{i}}-\hat{\boldsymbol{R}}_{\mathrm{CM}}-\sum_{\alpha=1}^{A-1} \omega_{\alpha}^{(j)} \hat{\boldsymbol{\rho}}_{\mathrm{R}_{\alpha}}^{(j)}\right|}+\hat{H}_{\mathrm{CM}+\mathrm{S}},
\end{aligned}
$$

where the indices $\mathrm{CM}$ and $\mathrm{R}$ refer to the atomic nucleus. Notice the tripartite interaction $\hat{H}_{\mathrm{e}+\mathrm{CM}+\mathrm{R}}$ in the above expression - this interaction has the origin in the term $\hat{V}_{\text {Coul }}^{\text {ep }}$, Eq. (4), while bearing in mind the relation:

$$
\hat{\boldsymbol{r}}_{\mathrm{p} j}=\hat{\boldsymbol{R}}_{\mathrm{CM}}+\sum_{\alpha=1}^{A-1} \omega_{\alpha}^{(j)} \hat{\boldsymbol{\rho}}_{\mathrm{R}_{\alpha}}^{(j)},
$$

that relates the position-observable of the $j$-th proton on the one, and of the position-observables for the nucleus center-of-mass and of the relative particles $\left(\omega_{\alpha}^{(j)}\right.$ represents the real coefficient), on the other side.

Effectively, this interaction couples the nucleus' CM and $\mathrm{R}$ subsystems as desired [5], thus formally fulfilling the necessary condition for $\mathrm{R}$ to act as CM's environment. However, this program breaks in its very beginning.
Actually, the Stern-Gerlach effect has also been observed for the hydrogen atom — as performed first by Phipps and Taylor [13]. The point is that the internal environment $\mathrm{R}$ simply does not exist for the hydrogen atom (for which the atomic number $A=1$, cf. Eq. (7)), and therefore, as a matter of principle, makes the system $\mathrm{R}$ unnecessary for the description of the Stern-Gerlach experiment.

Bearing in mind that we can not recognize the internal environment in the realistic models of atom, we are forced to conclude that the classical trajectories should be considered as physically unrealistic.

\section{Discussion}

Our conclusion on physical non-objectivity of classical trajectories stems from the minimal extension of the standard model [2] of the Stern-Gerlach experiment. Actually, relative to the standard model, Eq. (2), we only introduce the internal environment $\mathrm{R}$ as a necessary condition for the existence of the "classical trajectories". Performing the $a b$ initio analysis we point out that such a role of $\mathrm{R}$ can not be justified, thus positively emphasizing Eq. (3) as the physical state of the $C M+S$ system.

The alternative to our conclusion (of non-existence of classical trajectories) may follow from the alternative models of the experiment. E.g., one may quantize the magnetic field, in which case it may act as the quantum apparatus. However, we do not find such alternative(s) physically reasonable, and we find our conclusion as a definite description of the Stern-Gerlach experiment.

The tripartite interaction $\hat{H}_{\mathrm{e}+\mathrm{CM}+\mathrm{R}}$ may seem interesting as possibly raising the following dilemma: whether or not this internal interaction may play some role in the Stern-Gerlach experiment with the atoms - all the kinds of atoms but the hydrogen atom? Interestingly enough, as we show below, the interaction $\hat{H}_{\mathrm{e}+\mathrm{CM}+\mathrm{R}}$ is not expected observably to contribute to the Stern-Gerlach effect. So, our conclusion on nonexistance of classical trajectories bears the full generality - i.e. equally refers to all the kinds of the atomic species.

In order to justify this notion, we give the following analysis of the atomic subsystems. The adiabatic parameter $\kappa=m_{\mathrm{R}} E_{\mathrm{CM}} / m_{\mathrm{CM}} E_{\mathrm{R}}$ [14] for the nucleus $\mathrm{CM}$ and $\mathrm{R}$ subsystems gives the order of $A^{-1} 10^{-9}$, where $A$ is the atomic mass number, and therefore there is a very good adiabatic cut between the two systems, CM and R. On the other side, the semiclassical estimation of the interaction $\hat{H}_{\mathrm{e}+\mathrm{CM}+\mathrm{R}}$, Eq. (6), that can be written as $k Z^{2} \sum_{i, \alpha=1}^{Z}\left|\boldsymbol{r}_{\mathrm{e} i}-\boldsymbol{R}_{\mathrm{CM}}\right|^{-3}\left|\boldsymbol{\rho}_{\mathrm{R} \alpha}\right|^{2}$, gives the order of $Z^{2} 10^{-9} \mathrm{eV}$ for $\hat{H}_{\mathrm{e}+\mathrm{CM}+\mathrm{R}}$. Therefore, this interaction is much weaker than the self-energy $E_{\mathrm{CM}}$ of the CM system that is of the order of $10^{-2} \mathrm{eV}$. So, this interaction does not dominate the system's dynamics and one may apply the adiabatic approximation [14], i.e. to take the separable state $|\Psi\rangle_{\mathrm{CM}+\mathrm{S}}|\phi\rangle_{\mathrm{R}}$ as a very good approximation, where $|\Psi\rangle_{\mathrm{CM}+\mathrm{S}}$ is given by Eq. (3). 
While our conclusion offers some interesting observations and lessons for the foundations of quantum mechanics (quantum measurement, transition from quantum to classical [5], of the issue of the arrow of time [15] — to be presented elsewhere), it is also of interest for the foundations of the atomic nanofabrication as well.

In the context of the quantum measurement problem, our conclusion points out the active role of the screen in the spin measurement. Actually, as the classical information about the atomic trajectories appears only on the screen, the screen appears as a substantial element of the quantum measurement of spin. This observation strenghtens the possibility that the quantum measurement may not be complete without an act of the quantum particles detection.

The atomic nanofabrication procedures $[6,7,16$, $17,18]$ are typically expressed in the terms of classical trajectories for atoms. Needless to say, if literally understood, these phrases may leave an impression of the semiclassical character of the atomic center-of-mass degrees of freedom, and, consequently, that the manipulation with the atomic beams can (and may) be safely designed in the classical-physics manner.

However, as our study points out: the atomic spatial degrees of freedom - even for the different decompositions of the atom into subsystems - bear the full quantum mechanical potential. As the external (classical) magnetic field does not mutually couple CM and R systems, the Stern-Gerlach experiment must be described by the entangled state, Eq. (3). The entangled state, Eq. (3), provides the classically unknown possibilities for manipulating with the CM system by directly manipulating with the atomic spin system S. Certainly, this observation directly sets a specific recipe for manipulating the atomic beams in the realistic physical situations in the atomic nanofabrication procedures - that is our conclusion.

\section{Acknowledgments}

The work on this paper is financially supported by Ministry of Science Serbia under contract No 141016.

\section{References}

[1] W. Pauli, in: Handbuck der Physik, Ed. S. Flugge, Springer, Berlin 1985, p. 165.

[2] D. Bohm, Quantum Theory, Prentice Hall, New York 1989.

[3] T.R. Oliveira, A.O. Caldeira, Coherence and entanglement in a Stern-Gerlach experiment, arXiv:quant-ph/0608192v1, 24 Aug 2006.

[4] M. Nielsen, I. Chuang, Quantum Computation and Quantum Information, Cambridge, UK 2000.

[5] E. Joos, H.D. Zeh, C. Kiefer, D. Giulini, J. Kupsch, I.-O. Stamatescu, Decoherence and the Appearance of a Classical World in Quantum Theory, Springer, New York 2003.

[6] D. Meschede, H. Metcalf, J. Phys. D, Appl. Phys. 36 R17-R38 (2003).

[7] D. Meschede, Optics, Light and Lasers: The Practical Approach to Modern Photonics and Laser Physics, Wiley-VCH, Weinheim 2007.

[8] H.-P. Breuer, F. Petruccione, The Theory of Open Quantum Systems, Oxford University Press, New York 2007.

[9] M. Dugić, Eur. Phys. J. D 29, 173 (2004).

[10] R. Omnès, The Interpretation of Quantum Mechanics, Princeton University Press, Princeton 1994.

[11] A.O. Caldeira, A.J. Leggett, Ann. Phys. (NY) 149, 374 (1983).

[12] Y. Miroshnychenko, D. Schrader, S. Kuhr, W. Alt, I. Dotsenko, M. Khudaverdyan, A. Rauschenbeutel, D. Meschede, Optics Express 11, 3498 (2003).

[13] T.E. Phipps, J.B. Taylor, Phys. Rev. 29, 309 (1927).

[14] A. Messiah, Quantum Mechanics, North-Holand Publishing Company, Amsterdam 1976.

[15] H.D. Zeh, The Physical Basis of The Direction of Time, Springer-Verlag, 4-th Edition, Berlin 2001.

[16] R. Arun, J.Ch. Averbulch, T. Pfau, Phys. Rev. A 72 , 023417 (2005).

[17] F. Tantussi, A. Camposeo, M. Alderighi, N. Puccini, E. Andreoni, M. Allegrini, E. Arimondo, F. Fuso, Mat. Sci. Eng. C 27, 1418 (2007).

[18] Z. Cui, Nanofabrication - Principles, Capabilities and Limits, Springer, New York 2008. 